\title{
Water Vapor Emission From Rigid Mesoporous Materials during the Constant Drying Rate Period
}

\author{
T. Diaz Gonçalves,${ }^{1}$ V. Brito, ${ }^{1}$ and L. Pel ${ }^{2}$ \\ ${ }_{1}$ National Laboratory for Civil Engineering (LNEC), Materials Department, Lisbon, Portugal \\ 2Department of Applied Physics, Eindhoven University of Technology, Eindhoven, The Netherlands
}

\begin{abstract}
It has long been thought that the evaporation rate from mesoporous materials during the constant drying rate period (CDRP) is equal to that of a free-water surface, due to the presence of a liquid film covering the surface of the material. In this article we review several early articles and demonstrate that the experimental scrutiny this hypothesis has received is insufficient. Further, we report a set of evaporative drying experiments on eight building materials whose results also do not confirm such hypothesis. Indeed, the drying rate during the CDRP is not equal either among the tested materials or between these and the freewater surfaces. To explain the differences in drying rate, we have looked at the influence of surface texture and porosity. We have concluded that surface texture, which could increase the effective surface area of the materials, did not have a relevant effect on the CDRP drying rate. However, we have found a good correlation between the CDRP drying rate and capillary porosity. This is consistent with the hypothesis that drying occurs at the pore level during the CDRP. Further, it contradicts the suggestion that there is a film of water covering the surface of the materials during this period.
\end{abstract}

Keywords: Constant drying rate period; Drying rate; Heat and mass transfer; Pore network; Porous media 